\title{
Guillain-Barré syndrome associated with leptomeningeal enhancement following SARS-CoV-2 infection
}

\author{
Authors: Agustín Sancho-Saldaña, ${ }^{\mathrm{A}}$ Álvaro Lambea-Gil,, Jose Luis Capablo Liesa, ${ }^{\mathrm{A}}$ Maria Rosario Barrena Caballo, ${ }^{\mathrm{B}}$ \\ Maria Haddad Garay, ${ }^{\mathrm{C}}$ David Rivero Celada ${ }^{\mathrm{D}}$ and Marta Serrano-Ponz ${ }^{\mathrm{A}}$
}

\section{Introduction}

Patients with coronavirus disease 2019 (COVID-19) typically present with respiratory symptoms, but little is known about the disease's potential neurological complications.

We report a case of Guillain-Barré syndrome (GBS) following a severe acute respiratory syndrome coronavirus 2 (SARS-CoV-2) infection, in association with leptomeningeal enhancement.

\section{Case presentation}

A 56-year-old woman presented with recent unsteadiness and paraesthesia in both hands. Fifteen days earlier, she complained of fever, dry cough and shortness of breath. Her chest X-ray showed a lobar consolidation and PCR was positive for SARSCoV-2; she was admitted due to mild COVID-19 pneumonia.

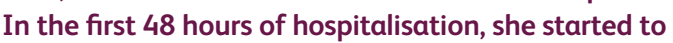
experience lumbar pain and weakness of the proximal lower extremities, progressing to bilateral facial nerve palsy, oropharyngeal weakness and severe proximal tetraparesis with cervical flexion 2/5 on the MRC scale. Full spine magnetic resonance imaging (MRI) showed a brainstem and cervical leptomeningeal enhancement. Analysis of cerebrospinal fluid (CSF) revealed albumin-cytological dissociation. Microbiological studies on CSF, including SARS-CoV-2, were negative. Nerve conduction studies were consistent with demyelinating neuropathy. She was treated with intravenous immunoglobulin, with significant neurological improvement noted over the next 2 weeks.

\section{Conclusion}

Leptomeningeal enhancement is an atypical feature in GBS, but could be a marker of its association with SARS-CoV-2 infection.

KEYWORDS: COVID-19, SARS-CoV-2, Guillain-Barré syndrome, leptomeningeal enhancement, coronavirus

DOI: $10.7861 /$ clinmed.2020-0213

Authors: Aconsultant in neurology, Hospital Universitario Miguel Servet, Zaragoza, Spain; ${ }^{\mathrm{B}}$ consultant in neuroradiology, Hospital Universitario Miguel Servet, Zaragoza, Spain; ' Consultant in clinical neurophysiology, Hospital Universitario Miguel Servet, Zaragoza, Spain; ${ }^{\text {D }}$ Consultant in neurosurgery, Hospital Universitario Miguel Servet, Zaragoza, Spain

\section{Introduction}

The outbreak of coronavirus disease 2019 (COVID-19), originating from Wuhan (China), is caused by severe acute respiratory syndrome coronavirus 2 (SARS-CoV-2), which is closely related to SARS-CoV-1 and Middle East respiratory syndrome coronavirus (MERS-CoV). It has spread rapidly around the world and has become pandemic. Patients' symptoms typically include non-productive cough, fever, fatigue and dyspnoea, causing pneumonia and severe acute respiratory syndrome (SARS). ${ }^{1}$ Although neurological manifestations such as hyposmia, dizziness or headache are increasingly recognised, there is little literature regarding neuromuscular complications.

We report a patient presenting with Guillain-Barré syndrome (GBS) following a SARS-CoV-2 respiratory infection.

\section{Case presentation}

A 56-year-old woman presented with recent unsteadiness and paraesthesia in both hands. Fifteen days earlier, she had reported fever, dry cough and shortness of breath that was controlled with symptomatic treatment. All relatives with whom she lived had the same symptoms, two being positive for SARS-CoV-2 and one of them dying due to severe respiratory infection in the weeks before. Her chest X-ray showed a lobar consolidation, and nasopharyngeal swab was positive for SARS-CoV-2 on PCR assay. She started treatment with hydroxychloroquine and azithromycin.

In the first 48 hours of hospitalisation, she developed lumbar pain and progressive proximal lower limb weakness. Physical examination at that time found normal cognition and cranial nerve function, but a mild proximal tetraparesis $4 / 5$ on the Medical Research Council (MRC) scale with global areflexia. Sensation to light touch, pinprick and proprioception were normal. There were no problems with sphincter control.

Due to the diagnostic uncertainty, given the limited knowledge related to the neurological complications of SARS-CoV-2, we requested magnetic resonance imaging (MRI) of her whole spine, which showed brainstem and cervical meningeal enhancement (Fig 1). Her cerebrospinal fluid (CSF) showed three leukocytes and protein of $0.86 \mathrm{~g} / \mathrm{L}$. Microbiological studies on CSF, including SARS$\mathrm{CoV}-2$, were negative. Antiganglioside antibodies were negative. We started intravenous immunoglobulin $2 \mathrm{~g} / \mathrm{kg}$ over 5 days. However, her condition progressed in the next 24 hours with bilateral facial nerve palsy, oropharyngeal weakness and severe 


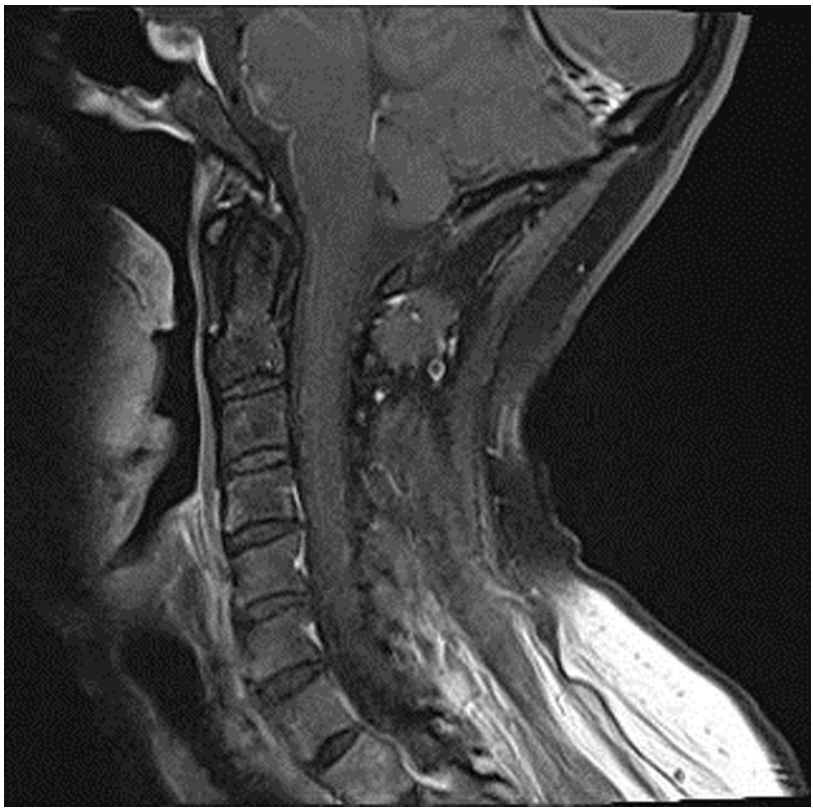

Fig 1. T1-weighted sagittal imaging after gadolinium, showing an anterior brainstem and cervical leptomeningeal enhancement.

proximal tetraparesis with cervical flexion $2 / 5$ on the MRC scale. She was transferred to the intensive care unit for 5 days due to the risk of respiratory insufficiency and began rehabilitation, not needing mechanical ventilation. She started recovering by day 7 after the onset of weakness. On her worst neurological examination, she had bilateral facial diplegia, dysphagia, tetraparesis $2 / 5$ proximal and $3 / 5$ distal on the MRC scale and global areflexia. There was no compromise of eye movements or ataxia.

Nerve conduction studies on day 11 showed delayed distal latencies and absent $\mathrm{F}$ waves, consistent with a demyelinating neuropathy.

\section{Discussion}

To date, there have been a very few reported cases of GBS associated with COVID-19. 2,3 Some of these followed a parainfective profile, raising the question of whether this association could be coincidental. GBS overlapping with Bickerstaff's brainstem encephalitis (BBE) was reported in association with MERS-CoV infection, ${ }^{4}$ but we have not found any case in association with SARS-CoV-1, where neuromuscular problems are considered to be, mostly, critical-illness polyneuropathy or myopathy.

We report a case of GBS following SARS-CoV-2 infection in a postinfective profile as reported in association with other viruses such as cytomegalovirus, Epstein-Barr virus, influenza A virus, Zika virus or hepatitis E virus. ${ }^{5}$
Contrast-enhanced MRI of spine is suggested as a supplementary diagnostic modality in diagnosing GBS, especially when the clinical and electrophysiological findings are equivocal. In this patient, the contrast-enhanced T1-weighted sequence suggested a subtle anterior cervical and brainstem leptomeningeal enhancement. In GBS, enhancement of spinal roots is not uncommon and indicates a breakdown of the blood-nerve barrier. However, diffuse leptomeningeal enhancement has recently been documented in paediatric GBS, spreading from the posterior fossa to the cauda equina. ${ }^{6}$ In a recent report, eight out of 13 SARS-CoV-2 infections with unexplained encephalopathy had leptomeningeal enhancement without cells in CSF.?

Some authors suggest that coronaviruses may be neurotropic and directly infect and damage motor neurons and peripheral nerves, as seen in some viral infections such as poliovirus, enterovirus D68 or cytomegalovirus. ${ }^{8}$ However, in this patient, the absence of cells and the negativity for SARS-CoV-2 in CSF allowed us to rule out this hypothesis.

Overall, this report supports the existence of a causal relationship between GBS and SARS-CoV-2 infection in a classic postinfective pattern, but more cases are necessary for better understanding of this potential association. The meningeal enhancement related to SARS-CoV-2 warrants further investigation.

\section{References}

1 Mao L, Wang M, Chen S et al. Neurological manifestations of hospitalized patients with COVID-19 in Wuhan, China: a retrospective case series study. SSRN Electron ] 2020 [Epub ahead of print]; https:// www.medrxiv.org/content/10.1101/2020.02.22.20026500v1.

2 Zhao H, Shan D, Zhou H, Lui J, Che S. Guillain-Barré syndrome associated with SARS-CoV-2 infection: causality or coincidence? Lancet Neurol 2020;19:383-4.

3 Toscano G, Palmerini F, Ravaglia S et al. Guillain-Barré syndrome associated with SARS-CoV-2. N Engl ] Med 2020 [Epub ahead of print]; https://doi.org/10.1056/NEJMc2009191.

$4 \mathrm{Kim} \mathrm{JE}, \mathrm{HeO} \mathrm{JH}, \mathrm{Kim} \mathrm{HO}$ et al. Neurological complications during treatment of Middle East respiratory syndrome. J Clin Neurol 2017;13:227-33.

5 Willison $\mathrm{HJ}$, Jacobs BC, van Doorn PA. Guillain-Barré syndrome. Lancet 2016;388:717-27.

6 Goldman N, Cuvellier J-C, Soto-Ares G, Vallée L. A post cytomegi alovirus Guillain-Barré syndrome, with atypical presentation, associated with arachnoiditis in a 10-month-old girl. Neuro Neurosurg 2018;1:1-2. https://doi.org/10.15761/NNS.1000108.

7 Helms J, Kremer S, Merdji $\mathrm{H}$ et al. Neurologic features in severe SARS-CoV-2 infection. N Engl ] Med 2020 [Epub ahead of print]; https://doi.org/10.1056/NEJMc2008597.

8 Wu Y, Xu X, Chen Z et al. Nervous system involvement after infection with COVID-19 and other coronaviruses. Brain Behav Immun 2020 [Epub ahead of print]; https://doi.org/10.1016/j. bbi.2020.03.031.

Address for correspondence: Dr Agustín Sancho-Saldaña, Servicio de Neurología, Hospital Universitario Miguel Servet, Paseo Isabel la Católica 1-3, 50009, Zaragoza, Spain. Email: agustinsanchosaldana@gmail.com 